\title{
ALTERAÇÕES NA BANDA ALFA DO ELETRENCEFALOGRAMA DURANTE IMAGÉTICA MOTORA VISUAL E CINESTÉSICA
}

\author{
Marcus Vinicius Stecklow', Antonio Fernando Catelli Infantosi', Maurício Cagy³
}

\begin{abstract}
RESUMO - Foi investigada a contribuição em potência na vizinhança do pico da banda alfa (BPA) do EEG durante imagética motora (IM). Sinais EEG (derivações occipitais e parietais) foram adquiridos em sujeitos destros (18-40 anos), durante repouso (ESP) e IM nas modalidades cinestésica (IMC) e visual (IMV), sendo 15 atletas de voleibol e 15 não-atletas. O Revised Movement Imagery Questionnaire não indicou diferenças entre grupos ou modalidades de IM; todavia os atletas imaginaram-se melhor que os não-atletas. Para ambos os grupos, a potência em BPA resultou menor em IM do que em ESP, sendo mais reduzida em IMC. Ativação cortical similar ocorreu em ambos os hemisférios de não-atletas e mais pronunciada no hemisfério esquerdo de atletas, principalmente durante IMC. Tais resultados sugerem que IM reduz a atividade de alfa de acordo com o conhecimento real da tarefa e a modalidade de IM.
\end{abstract}

PALAVRAS-CHAVE: imagética motora, banda alfa, EEG multicanal.

\section{Changes in the electroencephalogram alpha band during visual and kinesthetic motor imagery}

ABSTRACT - This study aims at statistically assessing the differences in alpha band power, particularly in the vicinity of the alpha peak (BPA), during motor imagery (MI). Multi-channel EEG (occipital and parietal regions) was acquired at rest condition (ESP) and MI kinesthetic (MIC) and visual (MIV) modalities from righthanded male subjects (18-40 years), 15 'athletes' (experienced volleyball players) and 15 'non-athletes'. The Revised Movement Imagery Questionnaire indicated no differences between groups or MI modalities, but athletes imagine themselves more clearly than non-athletes during MI. The power within BPA reduces in both groups, but greater in MIC than in MIV. The cortical activation was similar in both hemispheres of nonathletes but more pronounced in left hemisphere of athletes, mainly in MIC. The findings suggest that MI reduces alpha activity according to individual knowledge of real execution of motor task and MI modalities.

KEY WORDS: motor imagery, alpha band, multi-channel EEG.

A imagética motora (IM) é definida como um processo mental dinâmico no qual um sujeito simula uma tarefa motora sem que ocorra o movimento de quaisquer segmentos corporais associados a esta tarefa $a^{1,2}$. Em sessões de treinamento mental, a IM tem sido apontada como responsável pelo aumento de habilidades motoras em atividades esportivas como o voleibo $^{3}$ e críquete ${ }^{4}$, embora a prática real demonstre melhores resultados do que o treinamento mental $^{5}$. A IM tem origem basicamente visual e cinestésica ${ }^{6}$, sendo a modalidade visual correspondente à simulação mental de uma determinada tarefa motora como se estivesse observando um "vídeo mental", enquanto que, na IM cinestésica, o sujeito deve "sentir" como se o seu corpo estivesse em movimen- to, procurando obter sensações relacionadas às contrações musculares e da posição dos diversos segmentos corporais no espaço. Dentre os protocolos utilizados no estudo da IM, destacam-se os questionários, na maioria das vezes relacionados ao grau de nitidez ou percepção da IM. Estes apresentam a desvantagem de serem baseados em respostas subjetivas, como o Movement Imagery Questionnaire ${ }^{7}$. Outros protocolos incluem a coleta de sinais fisiológicos, tais como o eletrencefalograma (EEG) $)^{8,9}$ e/ou das freqüências cardíaca e respiratória, bem como potencial e resistência elétrica cutâneos ${ }^{3}$.

Os estudos relacionados às modalidades de IM têm indicado que algumas tarefas envolvem a informação de imagem e percepção, requerendo uma al-

Universidade Federal do Rio de Janeiro, Rio de Janeiro RJ, Brasil (UFRJ) e Universidade Federal Fluminense, Niterói RJ, Brasil (UFF): ${ }^{1}$ Educador Físico, M.Sc. em Engenharia Biomédica pela COPPE, UFRJ; ${ }^{2}$ Professor Titular do Programa de Engenharia Biomédica da COPPE, UFRJ; ${ }^{3}$ Professor Adjunto do Departamento de Epidemiologia e Bioestatística, UFF. Estudo financiado parcialmente pela CAPES e CNPq.

Recebido 4 Maio 2007, recebido na forma final 26 Julho 2007. Aceito 24 Agosto 2007. 
ternância na modalidade de IM ou a utilização de ambas $^{10}$. Embora o hemisfério esquerdo, especialmente o córtex parietal posterior, seja responsável pelo planejamento de movimentos ${ }^{11}$, a realização de IM em diferentes modalidades tem demonstrado ativação de áreas distintas do córtex, como já indicado em estudos com sujeitos doentes ${ }^{12}$ e sadios $^{13,14}$.

A utilização do EEG, particularmente a análise da banda alfa (8-13 Hz), tem-se mostrado importante ferramenta de análise do funcionamento cerebral, tanto em protocolos que utilizam a execução real de tarefas motoras ${ }^{15}$, quanto em $\mathrm{IM}^{8}$, sendo a magnitude da potência nesta banda do EEG inversamente relacionada ao nível de esforço mental. Em alguns estudos, a banda alfa tem sido dividida nas sub-bandas inferior (8-10 Hz), associada a níveis de atenção e esforço; e superior (11-13 Hz), que responde basicamente à codificação de estímulos e memória semântica ${ }^{16,17}$. Enquanto alguns autores têm indicado uma redução na potência da banda alfa durante $\mathrm{IM}^{8}$, outros encontraram resultados diferentes ${ }^{18}$. Tais resultados foram obtidos por meio de diferentes técnicas para análise do EEG, como potenciais relacionados a eventos ${ }^{14}$, análise espectral ${ }^{8}$ e de componentes independentes ${ }^{9}$. Na maioria dos estudos sobre IM envolvendo o emprego de EEG, são utilizadas tarefas motoras simples. A utilização de IM de tarefas de elevada complexidade de execução, tal como um movimento de ataque no voleibol, ainda carece de mais estudos.

O objetivo deste estudo foi avaliar alterações na banda alfa de regiões corticais distintas, durante imagética cinestésica e visual em populações com diferentes graus de vivência na execução real da tarefa acima indicada. A potência referente à banda na vizinhança do pico de alfa será estimada e avaliada estatisticamente, a partir da densidade espectral de potência (DEP) de sinais EEG durante IM em condições distintas.

\section{MÉTODO}

Foram estudados 33 voluntários do sexo masculino, destros, com faixa etária de 18 a 34 anos, subdivididos em dois grupos. O primeiro (GA), formado por 15 atletas de voleibol com média de idade $22,73( \pm 2,34)$ anos e o segundo, por 18 sujeitos com média de idade $27,27( \pm 4,06)$ anos sem experiência na prática desse desporto, denominados nãoatletas (GNA).

Os experimentos foram realizados no Laboratório de Mapeamento Cerebral e Integração Sensório Motora no Instituto de Psiquiatria e no Laboratório de Processamento de Imagens e Sinais do Programa de Engenharia Biomédica, ambos da Universidade Federal do Rio de Janeiro. Cada voluntário assinou termo de Consentimento Livre e Esclarecido e preencheu ficha de anamnese que, além dos dados pessoais, continha informação sobre o grau de experiência na prática de voleibol.
O Revised Movement Imagery Questionnaire (MIQ-R), que consiste na execução real, imagética visual e cinestésica de tarefas motoras simples foi aplicado a cada voluntário para determinar sua capacidade de realizar $\mathrm{IM}^{19}$. Como critério de exclusão, utilizou-se o escore menor que 15 para a soma dos valores em cada conjunto de tarefas, que resultou na exclusão de três não-atletas da casuística inicial. Os demais voluntários assistiram a um vídeo de 5 minutos com seqüências de ataque de voleibol de quadra, gravadas de diferentes posições para obter uma completa demonstração do movimento. Com os voluntários sentados confortavelmente, foram posicionados eletrodos de superfície do tipo $\mathrm{Ag} / \mathrm{AgCl}$ sobre o escalpo nas regiões occipitais e parietais homólogas ( $\mathrm{O}_{1}$ e $\mathrm{O}_{2}, \mathrm{P}_{3}$ e $\mathrm{P}_{4}$, segundo o Sistema Internacional 10-20), utilizando-se a referência média bi-auricular com terra em Fpz. Os sinais EEG, adquiridos por meio de um eletrencefalógrafo EMSA mod. BNT-36, foram submetidos à filtragem anti-aliasing de $100 \mathrm{~Hz}$, passa-altas de $0,1 \mathrm{~Hz}$ e notch de $60 \mathrm{~Hz}$ e, então, amostrados a $240 \mathrm{~Hz}$. Tais sinais foram coletados em três condições experimentais distintas: EEG espontâneo (ESP), imagética motora cinestésica (IMC) e visual (IMV). Em ESP (com contagem mental decrescente), durante 90 s precedendo IMC, a seguir, precedendo IMV e ao final do experimento.

Para cada uma das condições (IMC e IMV), foram adquiridos 30 segmentos de $7 \mathrm{~s}$ de EEG, cada um simulando mentalmente o movimento de ataque apresentado no vídeo (tarefa-alvo), intercalados aleatoriamente com a aquisição de vinte segmentos durante simulação mental de tarefa distratora (bater palmas sentado numa cadeira). O início de cada tarefa foi disparado por um sinal sonoro de tonalidade diferente (alvo ou distratora), precedido em $2 \mathrm{~s}$ de outro sinal sonoro, indicando que o voluntário deveria preparar-se para execução da IM. Antes de IMC e IMV, foi apresentada a descrição verbal do movimento de ataque de voleibol por meio de instruções previamente gravadas. Após cada condição, a escala do MIQ-R foi utilizada para mensurar a nitidez durante imagética, utilizando-se a avaliação subjetiva do voluntário.

Os primeiros oito segmentos não foram utilizados no processamento do sinal EEG de modo a se evitar possível processo de habituação, que implicaria não estacionariedade do sinal. Com vistas à rejeição de artefatos, utilizouse um algoritmo de remoção automática ${ }^{20,21}$, havendo ainda a opção de rejeição manual. Os 16 segmentos restantes de cada condição foram divididos em épocas de $5 \mathrm{~s}$ de duração com um total de 1200 amostras, sendo o início de cada uma sincronizada pelo sinal sonoro indicativo do início de cada IM. A seguir, a densidade espectral de potência (DEP) de cada época foi estimada utilizando-se Transformada Discreta de Fourier (DFT). Para aumentar a significância estatística desta estimativa, utilizou-se o periodograma de Bartlett $^{22}$, expresso por:

$$
\hat{P}_{B}(f)=\frac{1}{M} \sum_{m=0}^{M-1} \widetilde{P}_{x x}^{(m)}(f)
$$

onde $M$ é o número total de trechos e $\widetilde{P}_{x x}^{(m)}(f)$ é o espectro estimado do $m$-ésimo trecho (independentes e com igual duração). No presente estudo, cada época foi subdividida 


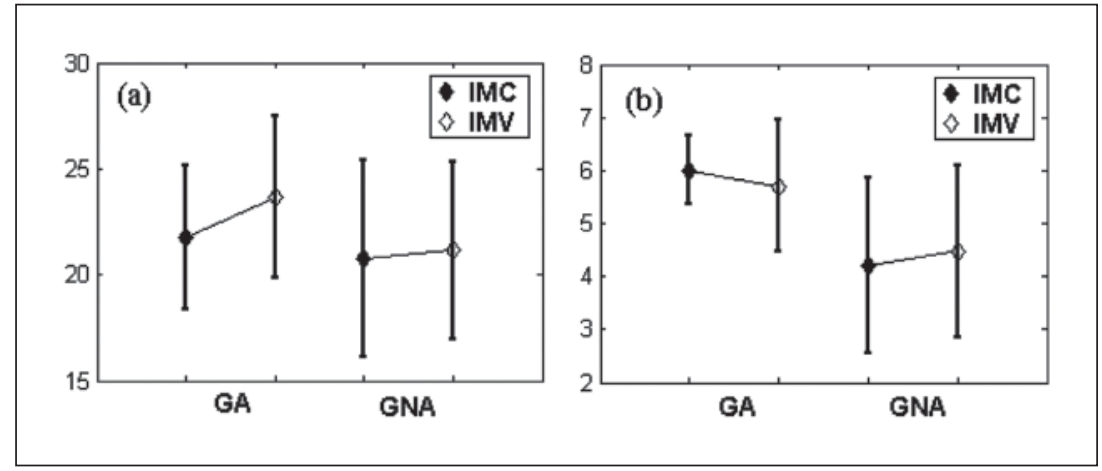

Fig 1. Valor médio ( $\bullet$ ) mais ou menos um desvio-padrão (segmento de reta) dos escores do (a) MIQ-R e da (b) nitidez da IM, nas modalidades cinestésica e visual para GA e GNA

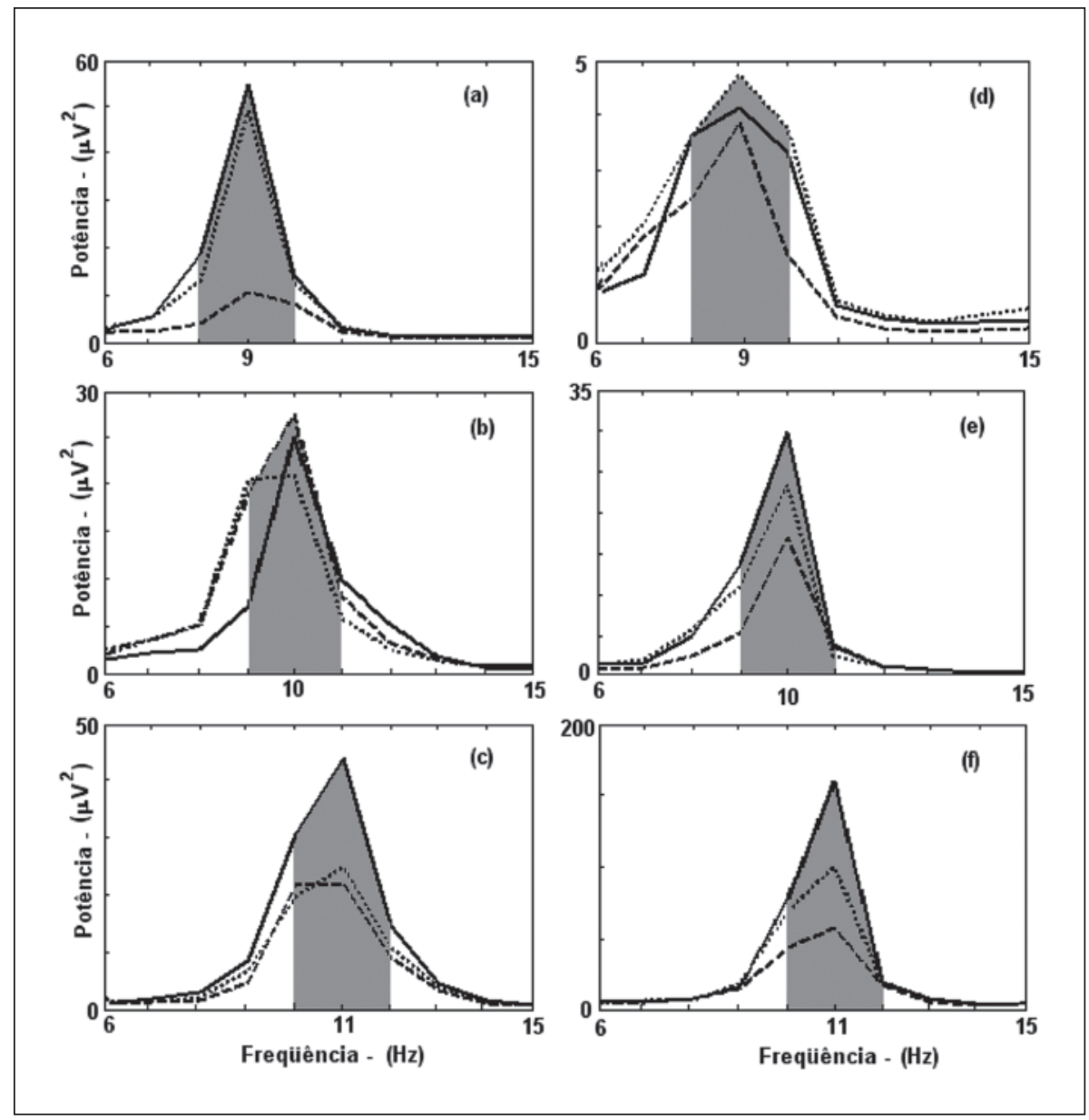

Fig 2. Estimativa espectral de potência do EEG da derivação O1, na faixa de 6 a $15 \mathrm{~Hz}$ e indicando BPA pela área em cinza, adquirido de atletas (a) \#15, (b) \#1, (c) \#2 e não-atletas (d) \#2, (e) \#7 e (f) \#9, durante imagética motora cinestésica (IMC: linha tracejada), visual (IMV: linha pontilhada) e atividade espontânea (ESP: linha cheia).

em $M=5$ trechos de $1 \mathrm{~s}$ de duração (resolução espectral de 1 $\mathrm{Hz}$ ) e, então, a DEP foi estimada.

Devido à variabilidade interindividual do pico de alfa (freqüência da máxima contribuição de potência na banda), uma faixa de $2 \mathrm{~Hz}$ centrada nesse pico (BPA) foi utilizada para estimativa da potência nesta banda. Para o cálculo da BPA, foi utilizado o método de integração trapezoidal, que consiste no cálculo da área limitada acima pelo espectro e aos lados pelos pontos que definem as freqüências limites da BPA.

O teste pareado de Wilcoxon foi aplicado na avaliação da significância estatística para a hipótese nula (Hom) de 
Tabela. Valores-p do teste pareado de Wilcoxon entre ESP, IMC e IMV para atletas e não-atletas em BPA.

\begin{tabular}{|c|c|c|c|c|c|c|}
\hline & \multicolumn{3}{|c|}{ Atletas } & \multicolumn{3}{|c|}{ Não-atletas } \\
\hline & ESP $\times$ IMC & ESP $x$ IMV & IMC x IMV & ESP $\times$ IMC & ESP $x$ IMV & IMC X IMV \\
\hline $\mathrm{O}_{1}$ & $0,0125^{*}$ & $0,0125^{*}$ & 0,8904 & $0,0151^{*}$ & $0,0215^{*}$ & 0,0554 \\
\hline $\mathrm{O}_{2}$ & 0,0946 & 0,2293 & 0,7197 & $0,0215^{*}$ & 0,3591 & 0,0833 \\
\hline$P_{3}$ & $0,0043^{*}$ & 0,3383 & 0,1514 & 0,0479 * & 0,2293 & $0,0479 *$ \\
\hline $\mathrm{P}_{4}$ & 0,0637 & 0,6788 & 0,1688 & $0,0479 *$ & 0,1876 & 0,1514 \\
\hline
\end{tabular}

(*) valor $\mathrm{p}<0,05$.

igualdade entre as medianas da contribuição média de potência em BPA nas diferentes condições experimentais (ESP, IMC e IMV) duas a duas. Os escores médios do MIQ-R (escalas visual e cinestésica) foram analisados usando-se o teste- $t$ de Student simples na comparação entre os grupos e pareado na comparação entre perspectivas dentro de cada grupo. O mesmo procedimento foi feito na análise dos graus de nitidez das imagens mentais formadas durante IMC e IMV. Foi aceito o nível de significancia de $5 \%$.

\section{RESULTADOS}

$\mathrm{Na}$ análise intragrupos do MIQ-R, o teste- $t$ de Student pareado indicou não ser possível rejeitar a hipótese nula entre os escores médios de IMV e IMC tanto para GA $(p=0,22)$ quanto para GNA $(p=0,84)$. Na comparação intergrupos para cada modalidade, também não houve rejeição da hipótese nula, seja para IMC $(p=0,53)$, seja para IMV $(p=0,10)$. A Figura $1 A$ indica os valores médios e a variabilidade dos escores do MIQ-R para ambos os grupos nas duas modalidades de IM.

A análise intragrupos da nitidez da IM do ataque de voleibol indicou não haver diferença significativa entre os escores médios de IMC e IMV tanto pra GA $(p=0,25)$ quanto para GNA $(p=0,63)$. Entretanto, a análise intergrupos (teste-t não pareado) demonstrou que os atletas obtiveram imagens mais nítidas que os não-atletas (Figura 1B) tanto durante IMC $(p<<0,05)$ quanto IMV $(p=0,04)$.

A Figura 2 ilustra a diferença interindividual da freqüência do pico de alfa entre voluntários de um mesmo grupo para o EEG da derivação O1, implicando em valores limítrofes distintos para a BPA. Nestes exemplos, nota-se que o pico de alfa varia de 9 a $11 \mathrm{~Hz}$, tanto para atletas quanto para não-atletas. Além disso, a contribuição relativa de potência também varia entre protocolos experimentais distintos. Assim, por exemplo, para os não-atletas, tal contribuição mostrou-se geralmente superior para a imagética motora visual (IMV) do que para a cinestésica (IMC).

A utilização do teste pareado de Wilcoxon (Tabela) permitiu verificar que ocorreram diferenças significativas $(p<5 \%)$ na potência de BPA entre ESP e IMC para todas as derivações em GNA, enquanto tal resul- tado ocorreu apenas nos sítios localizados no hemisfério esquerdo ( $\mathrm{O}_{1}$ e $\mathrm{P}_{3}$ ) de GA. Entre ESP e IMV, foi observada diferença significativa apenas na derivação O1 para ambos os grupos. Finalmente, na comparação entre IMC e IMV, apenas a derivação P3 apresentou diferença significativa.

\section{DISCUSSÃO}

Embora os escores médios para IM cinestésica e visual sejam ligeiramente maiores para os atletas, ambos os grupos apresentaram valores similares. As tarefas motoras utilizadas no MIQ-R, apesar da simples execução, não são treinadas ou utilizadas sistematicamente por qualquer dos grupos em suas atividades cotidianas. Durante IM do movimento de ataque de voleibol, entretanto, os atletas conseguiram imaginar tal movimento com mais nitidez que os não-atletas. Este resultado demonstra que a IM está intimamente relacionada com o processo de aprendizagem e planejamento de movimentos voluntários. Porém, não foi possível afirmar que alguma das modalidades de IM (cinestésica ou visual) seja mais fácil de ser executada se o indivíduo possuisse experiência prévia na execução real da tarefa.

A realização de IM em modalidades diferentes resultou na ativação de áreas distintas do córtex, com predominância do hemisfério esquerdo nos atletas, fato não observado nos não-atletas. Estes resultados, embora confirmem a participação do córtex parietal posterior do hemisfério esquerdo no planejamento e simulação de tarefas mentais ${ }^{11}$, indicam também que tal lateralização ocorre de acordo com o conhecimento prévio de execução da tarefa imaginada. Além desse aspecto, outra informação relevante consiste na diferenciação na ativação cortical de acordo com a modalidade de IM utilizada, a qual já foi demonstrada com indivíduos doentes ${ }^{12}$ e sadios ${ }^{13}$. Ambos os trabalhos, porém, utilizaram paradigmas desconhecidos para todos os sujeitos, diferentemente do nosso estudo, no qual os atletas possuíam conhecimento prévio na execução real da tarefa imaginada, e em 
que se procurou não apenas analisar a modalidade de IM, mas também o grau de conhecimento prévio na execução real da tarefa a ser imaginada.

A magnitude da potência na banda alfa do EEG tem sido inversamente associada ao grau de esforço cognitivo por uma determinada região cortical, podendo ocorrer aumento de tal magnitude em áreas não ativas, o que indica seletividade na utilização das redes neuronais durante o processamento da informação. A divisão da banda alfa em sub-bandas, conforme estudos anteriores ${ }^{8,16}$, não leva em conta a morfologia do espectro desta banda e a variabilidade interindividual existente; sendo assim, ao utilizarmos apenas a magnitude da potência ao redor do pico, pôde-se reduzir tal variabilidade interindividual na morfologia do espectro nesta banda do sinal de EEG, a despeito da perda de informação nas demais freqüências que compõem a banda alfa.

O presente estudo evidencia que a IM acarreta uma diferenciação na ativação cortical de acordo com o grau de vivência real desta tarefa. Enquanto, nos não-atletas, ocorre ativação em ambos os hemisférios, para os atletas, essa ativação é maior no hemisfério esquerdo. Salienta-se ainda que, além de estar associado ao processamento verbal, o hemisfério esquerdo também estaria ativo, especialmente na região do córtex parietal posterior, durante o planejamento de movimentos por IM ${ }^{11}$. Este resultado pode indicar que a simulação de tarefas complexas por indivíduos que não têm experiência prévia em sua execução demanda maior esforço cognitivo, conforme afirmou ${ }^{8}$, acarretando a ativação de regiões corticais do hemisfério direito para auxiliar o processo de aprendizagem.

Outro resultado relevante deste estudo concerne à diferença na ativação cortical de acordo com a modalidade de imagética executada. Durante IM cinestésica, ocorreram diferenças significativas em maior número de regiões corticais, especialmente nos nãoatletas. Esta ativação distinta do cérebro para modalidades diferentes de IM confirma outros achados ${ }^{12,16}$.

Em conclusão, os resultados do presente estudo demonstram a associação da imagética motora com o processo de planejamento e memorização de movimentos voluntários bem como a diferenciação na atividade neuronal, por meio da potência na banda de máxima contribuição em alfa, de acordo com o grau de conhecimento prévio na execução real da tarefa imaginada e com a modalidade de imagética motora utilizada. Em indivíduos com pouca vivência na tarefa motora imaginada, ambos os hemisférios apresentaram ativação pela imagética, enquanto que isto ocorreu apenas no hemisfério esquerdo nos sujeitos com experiência na prática real da tarefa.

\section{REFERÊNCIAS}

1. Decety J, Ingvar DH. Brain structures participating in mental simulation of motor behavior: a neuropsychological interpretation. Acta Psychol 1990;73:13-34.

2. Gentili R, Papaxanthis C, Pozzo T. Improvement and generalization of arm motor performance through motor imagery practice. Neuroscience $2006 ; 137: 761-772$.

3. Roure R, Collet C, Deschaumes-Molinaro C, Delhomme G, Dittmar A, Vernet-Maury E. Imagery quality estimated by autonomic response is correlated to sporting performance enhancement. Physiol Behav 1999;66:63-72.

4. Thewell RC, Maynard IW. The effects of a mental skills package on 'repeatable good performance' in cricketers. Psychol Sport Exerc 2003;4:377-396.

5. Ranganathan VK, Sieminow V, Liu, JZ, Saghal V, Yue, GH. From mental power to muscle power - gaining strength by using the mind. Neuropsychologia 2004;42:944-956.

6. Rodrigues EC, Imbiriba LA, Leite GR, Magalhães J, Volchan E, Vargas, CD. Efeito da estratégia de simulação mental sobre o controle postural. Rev Bras Psiquiatr 2003;25:33-35.

7. Hall C, Pongrac J, Buckolze E. The measurement of imagery ability. Hum Movement Sci 1985;4:107-118.

8. Cremades JG. The effects of imagery perspective as a function of skill level on alpha activity. Int J Psychophysiol 2002;43:261-271.

9. Naeem M, Brunner C, Leeb R, Graimann B, Pfustscheller G. Seperability of four-class motor imagery data using independent component analysis. J Neural Eng 2006;3:208-216.

10. Callow N, Hardy L. The relationship between the use of kinaesthetic imagery and different visual imagery perspectives. J Sport Sci 2004;22:167-177.

11. Rushworth MFS, Nixon PD, Renowden S, Wade DT, Passinghan RE. Left parietal cortex and motor attention. Neuropsychologia 1997;35:1261-1273.

12. Sirigu A, Duhamel JR. Motor and visual imagery as two complementary and neurally dissociable mental processes. J Cognitive Neurosci 2001;13:910-919.

13. Solodkin A, Hlustik P, Chen EE, Small SL. Fine modulation in network activation during motor execution and motor imagery. Cereb Cortex 2004;14:1246-1255.

14. Neuper C, Scherer R, Reiner M, Pfurtscheller G. Imagery of motor actions: Differential effects of kinesthetic and visual-motor mode of imagery in single-trial EEG. Cogn Brain Res 2005;25:668-677.

15. Cunha M, Bastos VH, Veiga H et al. Alterações na distribuição da potência cortical em função da consolidação da memória no aprendizado de datilografia. Arq Neuropsiquiatr 2004;62:662-668.

16. Jausovec N, Jausovec K. EEG activity during the performance of complex mental problems. Intl J Psychophysiol 2000;30:73-88.

17. Klimesch W, Pfurtscheller G, Schimke H. Pre- and post-stimulus processes in category judgement tasks as measured by event-related desynchronization (ERD). J Psychophysiol 1992;6:185-204.

18. Marks DF, Isaac AR. Topographical distribution of EEG activity accompanying visual and motor imagery in vivid and non-vivid imagers. Brit J Psychol 1995;86:271-282.

19. Hall CR, Martin KA. Measuring movement imagery abilities: A revision of movement imagery questionnaire. J Ment Imagery 1997;21:143-154.

20. Tierra-Criollo CJ, Infantosi AFC. Low-frequency oscillations in human tibial somatosensory evoked potentials. Arq Neuropsiquiatr 2006;64:402-406.

21. Infantosi AFC, Melges DB, Tierra-Criollo CJ. Use of magnitude-squared coherence to identify the maximum driving response band of the somatosensory evoked potential. Braz J Med Biol Res 2006;39:1593-1603.

22. Shiavi R. Introduction to applied statistical signal analysis. 2 Ed. Nashville: Academic Press, 1999. 